\title{
Synthesis of Boronocysteine
}

Samantha M. Gibson, Derek Macmillan and Tom D. Sheppard*

Department of Chemistry, University College London, Christopher Ingold Laboratories, 20 Gordon St, London, WC1H OAJ, UK

tom.sheppard@ucl.ac.uk

\section{Table of Contents}

1. General Experimental Information

2. Experimental procedures

3. ${ }^{1} \mathrm{H}$ and ${ }^{13} \mathrm{C}$ NMR spectra 10

4. References 


\section{General Experimental Procedures}

All solvents and chemicals were used as received. Column chromatography was carried out using either Merck Geduran Si 60 (40-63 $\mu \mathrm{m})$ silica gel or a Biotage purification system using Biotage or Grace silica columns. Analytical thin layer chromatography was carried out using Merck TLC Silica Gel $60 \mathrm{~F}_{254}$ aluminium-backed plates. Components were visualised using combinations of ultra-violet lights, potassium permanganate or ninhydrin. Proton magnetic resonance spectra ( ${ }^{1} \mathrm{H}$ NMR) were recorded at 400,500 or $600 \mathrm{MHz}$ on a Bruker Avance spectrometer and are reported as follows: chemical shift $\delta$ in ppm (number of protons, multiplicity, coupling constant $J$ in $\mathrm{Hz}$, assignment). The solvent used was deuterated chloroform or deuterated methanol unless stated otherwise. Residual protic solvent was used as the internal reference, setting $\mathrm{CDCl}_{3}$ to $\delta 7.26$ and $\mathrm{MeOD}-d_{4}$ to 3.31. The following abbreviations are used: $\mathrm{s}$, singlet; $\mathrm{d}$, doublet; $\mathrm{t}$, triplet; $\mathrm{q}$, quartet; $\mathrm{m}$, mulitplet; br, broad or a combination of these. Carbon magnetic resonance spectra $\left({ }^{13} \mathrm{C}\right.$ NMR) were recorded at 100,125 or $150 \mathrm{MHz}$ on a Bruker Avance spectrometer using deuterated chloroform or deuterated methanol and using the central reference of $\mathrm{CDCl}_{3}$ to $\delta 77.0$ or MeOD- $d_{4}$ to $\delta 49.15$ as the internal standard. Mass Spectrometry data were collected on either TOF or magnetic sector analysers either at the Department of Chemistry, University College London or at the EPSRC UK National Mass Spectroscopy Facility at Swansea University. The ionization method is reported in the experimental data. 
(2,2-Diethoxyethyl)(4-methoxybenzyl)sulfane (3b) ${ }^{1,2}$<smiles>CCOC(CSCc1ccc(OC)cc1)OCC</smiles>

To a stirred solution of bromoacetaldehyde diethyl acetal $(3.760 \mathrm{~mL}, 25 \mathrm{mmol})$ in EtOH $(110 \mathrm{~mL})$ and $\mathrm{NaOH}(1 \mathrm{M}, 50 \mathrm{~mL})$ at $0{ }^{\circ} \mathrm{C}$ was added para-methoxybenzyl mercaptan (3.831 $\mathrm{mL}, 27.5 \mathrm{mmol})$ dropwise and the reaction stirred for 2 days. A precipitate formed which was removed by filtration and the filtrate reduced in vacuo. The residue was dissolved in ether $(25 \mathrm{~mL})$ and washed with $\mathrm{H}_{2} \mathrm{O}(25 \mathrm{~mL})$ and then brine $(20 \mathrm{~mL})$. The organic layers were dried $\left(\mathrm{MgSO}_{4}\right)$ and reduced in vacuo to give $3 \mathbf{b}$ as a light yellow oil (3.56 g, $13.2 \mathrm{mmol}, 52 \%) ; \mathbf{R}_{\mathbf{f}} 0.28$ (3:1 $\left.\mathrm{CH}_{2} \mathrm{Cl}_{2}: \mathrm{EtOAc}\right) ;{ }^{1} \mathbf{H} \mathbf{N M R}\left(600 \mathrm{MHz}, \mathrm{CDCl}_{3}\right) \delta 7.25$ $(2 \mathrm{H}, \mathrm{d}, J=8.5, \mathrm{ArH}), 6.83(2 \mathrm{H}, \mathrm{d}, J=8.5, \mathrm{ArH}), 4.54(1 \mathrm{H}, \mathrm{t}, \mathrm{J}=5.5, \mathrm{CH}), 3.77(3 \mathrm{H}, \mathrm{s}$, $\left.\mathrm{OCH}_{3}\right), 3.74\left(2 \mathrm{H}, \mathrm{s}, \mathrm{ArCH}_{2}\right), 3.64\left(2 \mathrm{H}, \mathrm{dq}, \mathrm{J}=9.3,7.1,2 \times \mathrm{OCH}_{2}\right), 3.51(2 \mathrm{H}, \mathrm{dq}, \mathrm{J}=9.3$, $\left.7.1,2 \times \mathrm{OCH}_{2}\right) 2.57\left(2 \mathrm{H}, \mathrm{d}, \mathrm{J}=5.5, \mathrm{SCH}_{2} \mathrm{CH}\right), 1.21\left(6 \mathrm{H}, \mathrm{t}, \mathrm{J}=7.1,2 \times \mathrm{CH}_{2} \mathrm{CH}_{3}\right) ;{ }^{13} \mathbf{C ~ N M R}$ $\delta\left(150 \mathrm{MHz}, \mathrm{CDCl}_{3}\right) \delta 158.7,130.5,130.3,114.0,103.5,62.3,55.4,36.2,34.0,15.5$; IR Umax 2973 (C-H), 1609 (Ar), 1509 (Ar), 1300 (C-O), 1244 (ArO-C).

\section{2-((4-Methoxybenzyl)thio)acetaldehyde (4) ${ }^{2}$}<smiles>COc1ccc(CSCC=O)cc1</smiles>

Dilute $\mathrm{HCl}(2 \mathrm{M}, 15 \mathrm{~mL})$ was added to $3 \mathbf{b}(1.9336 \mathrm{~g}, 7.158 \mathrm{mmol})$ in acetone $(15 \mathrm{~mL})$ and the reaction stirred overnight. The solution was extracted (EtOAc, $2 \times 10 \mathrm{~mL}$ ) and the organic layer washed with $\mathrm{H}_{2} \mathrm{O}(10 \mathrm{~mL})$ and then brine $(10 \mathrm{~mL})$. The organic layer was dried $\left(\mathrm{MgSO}_{4}\right)$, filtered, and reduced in vacuo to give 20 as a colourless oil $(1.39 \mathrm{~g}, 7.08$ mmol, 99\%). Rf 0.4 (9:1 petrol:EtOAc); ${ }^{1} \mathrm{H}$ NMR $\left(600 \mathrm{MHz}, \mathrm{CDCl}_{3}\right) \delta 9.40(1 \mathrm{H}, \mathrm{t}, J=3.5$, $\mathrm{CHO}), 7.21(2 \mathrm{H}, \mathrm{d}, J=8.6, \mathrm{ArH}), 6.85(2 \mathrm{H}, \mathrm{d}, J=8.6, \mathrm{ArH}), 3.79\left(3 \mathrm{H}, \mathrm{s}, \mathrm{OCH}_{3}\right), 3.58(2 \mathrm{H}$, $\left.\mathrm{s}, \mathrm{ArCH}_{2}\right), 3.07\left(2 \mathrm{H}, \mathrm{d}, J=3.5, \mathrm{CH}_{2} \mathrm{CHO}\right) ;{ }^{13} \mathrm{C}$ NMR $\left(150 \mathrm{MHz}, \mathrm{CDCl}_{3}\right) \delta$ 194.1, 159.0, 130.5, 128.9, 114.2, 55.4, 40.3, 35.1; IR $v_{\max } 3006(\mathrm{C}-\mathrm{H}), 2834(\mathrm{C}(\mathrm{O})-\mathrm{H}), 1714(\mathrm{C}=\mathrm{O})$, 1609, 1509, 1301, 1241, 1173, 1028. 
<smiles>COc1ccc(CSC/C=N/S(=O)CC(C)C)cc1</smiles>

Copper(II) Sulfate $(1.27 \mathrm{~g}, 7.94 \mathrm{mmol})$ and aldehyde $4(779 \mathrm{mg}, 3.97 \mathrm{mmol}, 1.1 \mathrm{eq})$ were added to a solution of ( \pm )-tert-butyl sulfonamide $\left(438 \mathrm{mg}, 3.61 \mathrm{mmol}\right.$ ) in anhydrous $\mathrm{CH}_{2} \mathrm{Cl}_{2}$ $(7.2 \mathrm{~mL})$. The reaction was stirred at room temperature for $18 \mathrm{~h}$, before filtering through Celite. The solvents were removed in vacuo and the residue obtained was purified by column chromatography to give $\mathbf{5}$ as an orange oil ( $865 \mathrm{mg}, 2.89 \mathrm{mmol}, 80 \%)$. ${ }^{1} \mathbf{H}$ NMR $\left(\mathrm{CDCl}_{3}, 600 \mathrm{MHz}\right) \delta 7.98(1 \mathrm{H}, \mathrm{t}, J=5.6, \mathrm{NCH}), 7.23(2 \mathrm{H}, \mathrm{d}, J=6.5, \mathrm{ArH}), 6.85(2 \mathrm{H}, \mathrm{d}, J=$ 6.5, $\mathrm{ArH}), 3.79\left(3 \mathrm{H}, \mathrm{s}, \mathrm{OCH}_{3}\right), 3.66\left(2 \mathrm{H}, \mathrm{s}, \mathrm{ArCH}_{2}\right), 3.35(1 \mathrm{H}, \mathrm{dd}, J=14.3,6.0,1 \times$ $\left.\mathrm{SCH}_{2} \mathrm{CH}\right), 3.31\left(1 \mathrm{H}, \mathrm{dd}, J=14.3,5.3,1 \times \mathrm{SCH}_{2} \mathrm{CH}\right), 1.22(9 \mathrm{H}, \mathrm{s}, t \mathrm{Bu}) ;{ }^{13} \mathrm{C} \mathrm{NMR}\left(\mathrm{CDCl}_{3}\right.$, $150 \mathrm{MHz}) \delta 164.2,158.9,130.3,129.2,114.1,57.0,55.4,35.02,34.3,22.5$; LRMS (Cl) $420(100 \%), 300\left(37 \%,[\mathrm{M}+\mathrm{H}]^{+}\right), 240(30 \%), 195\left(32 \%,[\mathrm{M}-\mathrm{SOtBu}]^{+}\right), 121\left(88 \%, \mathrm{PMB}^{+}\right)$; HRMS Found 300.10877, $\mathrm{C}_{14} \mathrm{H}_{22} \mathrm{NO}_{2} \mathrm{~S}_{2}$ requires 300.10865; IR $v_{\max }\left(\mathrm{film} / \mathrm{cm}^{-1}\right) 2958$ (C$H), 1609(\mathrm{C}=\mathrm{C}), 1510(\mathrm{C}=\mathrm{N}), 1458(\mathrm{C}=\mathrm{C}), 1083(\mathrm{~S}=\mathrm{O})$.

$N$-(2-((4-Methoxybenzyl)thio)-1-(4,4,5,5-tetramethyl-1,3,2-dioxaborolan-2-yl)ethyl)-2methylpropane-2-sulfinamide (6)

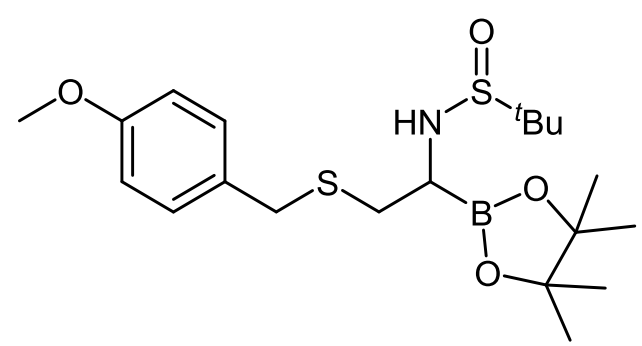

Using flame dried glassware under an argon atmosphere, $\mathrm{CuCl}(38.4 \mathrm{mg}, 0.388 \mathrm{mmol})$,

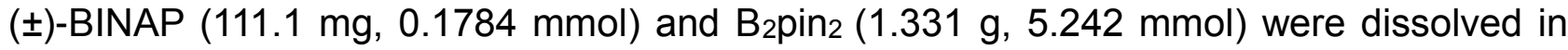
anhydrous THF ( $4 \mathrm{~mL})$. KOtBu (1 M in THF, $1.4 \mathrm{~mL}, 1.4 \mathrm{mmol}$ ) was added whilst stirring at room temperature. After $10 \mathrm{~min}$, the reaction was cooled to $-20{ }^{\circ} \mathrm{C}$ and aldehyde 5 $(1.0338 \mathrm{~g}, 3.4522 \mathrm{mmol})$ was added followed by $\mathrm{MeOH}(300 \mu \mathrm{L}, 7.41 \mathrm{mmol})$ and the reaction stirred overnight. The solvent was removed in vacuo and the resultant oil purified by flash column chromatography using EtOAc in $\mathrm{CH}_{2} \mathrm{Cl}_{2}(20 \rightarrow 35 \%)$ to give 6 as an 
orange oil (878 mg, $2.06 \mathrm{mmol}, 60 \%) .{ }^{1} \mathrm{H}$ NMR $\left(\mathrm{CDCl}_{3}, 600 \mathrm{MHz}\right) \delta 7.24(2 \mathrm{H}, \mathrm{d}, J=8.6$, $\mathrm{ArH}), 6.82(2 \mathrm{H}, \mathrm{d}, J=8.6, \mathrm{ArH}), 3.78\left(3 \mathrm{H}, \mathrm{s}, \mathrm{OCH}_{3}\right), 3.71(1 \mathrm{H}, \mathrm{d}, J=5.6, \mathrm{NH}), 3.69(2 \mathrm{H}, \mathrm{s}$, $\left.\mathrm{ArCH}_{2} \mathrm{~S}\right), 3.22-3.17(1 \mathrm{H}, \mathrm{m}, \mathrm{CHB}), 2.77\left(1 \mathrm{H}, \mathrm{dd}, J=13.4,6.3,1 \times \mathrm{SCH}_{2} \mathrm{CH}\right), 2.72(1 \mathrm{H}$, dd, $\left.J=13.4,7.9,1 \times \mathrm{SCH}_{2} \mathrm{CH}\right), 1.25\left(\mathrm{~s}, 6 \mathrm{H}, 2 \times\right.$ pinacol- $\left.\mathrm{CH}_{3}\right), 1.23(\mathrm{~s}, 9 \mathrm{H}, t \mathrm{Bu}), 1.20(\mathrm{~s}, 6 \mathrm{H}, 2$ $\times$ pinacol- $\left.\mathrm{CH}_{3}\right) ;{ }^{13} \mathrm{C} \mathrm{NMR}\left(\mathrm{CDCl}_{3}, 150 \mathrm{MHz}\right) \delta 158.7,130.1,130.0,114.0,84.3,56.2,55.3$, 41.3 (br), 35.2, 34.6, 25.0, 24.9, 22.6; LRMS (Cl) $428\left(41 \%,[\mathrm{M}+\mathrm{H}]^{+}\right), 371(18 \%$, $\left.[\mathrm{M}-t \mathrm{Bu}]^{+}\right), 322\left(38 \%,\left[\mathrm{M}-\mathrm{SO} \mathrm{BBu}^{+}\right), 121\left(100 \%, \mathrm{PMB}^{+}\right) ;\right.$HRMS Found 428.2095, $\mathrm{C}_{20} \mathrm{H}_{34} \mathrm{BNO}_{4} \mathrm{~S}_{2}$ requires 428.2095; IR $v_{\max }\left(\right.$ film/cm $\left.{ }^{-1}\right) 2977$ (C-H), 2930 (C-H), 1609 (C=C), $1511(\mathrm{C}=\mathrm{C}), 1544(\mathrm{C}=\mathrm{C}), 1369(\mathrm{~B}-\mathrm{O})$.

\section{2-((4-Methoxybenzyl)thio)-1-(4,4,5,5-tetramethyl-1,3,2-dioxaborolan-2-yl)ethanamine} hydrochloride (7)

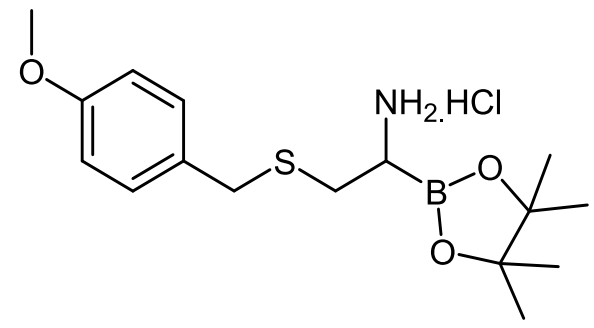

A solution of $\mathrm{HCl}$ in dioxane $(4 \mathrm{M}, 585 \mu \mathrm{L}, 2.34 \mathrm{mmol})$ was added to $6(99.7 \mathrm{mg}, 0.233$ $\mathrm{mmol}$ ) dissolved in anhydrous $\mathrm{MeOH}(3 \mathrm{~mL})$ to give a pale yellow solution. The reaction was stirred for $3 \mathrm{~h}$. The solvent was removed in vacuo to give an orange residue (75.3 $\mathrm{mg})$. The residue was washed with ether, sonicated, and centrifuged to give 7 as a light brown solid (68 mg, $0.190 \mathrm{mmol}, 82 \%$ ); mp 166-168 ${ }^{\circ} \mathrm{C}$ ). ${ }^{1} \mathrm{H}$ NMR (MeOD- $\left.d_{4}, 400 \mathrm{MHz}\right) \delta$ $7.29(2 \mathrm{H}, \mathrm{d}, J=8.7, \mathrm{ArH}), 6.89(2 \mathrm{H}, \mathrm{d}, J=8.7, \mathrm{ArH}) 3.80\left(3 \mathrm{H}, \mathrm{s}, \mathrm{OCH}_{3}\right), 3.79(2 \mathrm{H}, \mathrm{s}$, $\left.\mathrm{ArCH}_{2}\right), 3.01(1 \mathrm{H}, \mathrm{dd}, J=8.7,4.7, \mathrm{CHB}), 2.85\left(1 \mathrm{H}, \mathrm{dd}, J=14.3,4.8,1 \times \mathrm{CH}_{2} \mathrm{CH}\right), 2.73$ $\left(1 \mathrm{H}, \mathrm{dd}, J=14.3,8.8,1 \times \mathrm{CH}_{2} \mathrm{CH}\right), 1.33\left(12 \mathrm{H}, \mathrm{s}, 4 \times \mathrm{CH}_{3}\right) ;{ }^{13} \mathrm{C}$ NMR $\left(\mathrm{MeOD}-d_{4}, 100 \mathrm{MHz}\right)$ $\delta$ 159.1, 129.9, 129.5, 113.7, 85.5, 74.4, 54.5, 35.1, 30.4, 23.8, 23.7; LRMS (Cl) 323 $\left(100 \%,[\mathrm{M}+\mathrm{H}]^{+}\right), 198\left(18 \%,[\mathrm{M}-\mathrm{Bpin}]^{+}\right)$; HRMS Found 323.18359, $\mathrm{C}_{16} \mathrm{H}_{27} \mathrm{BNO}_{3} \mathrm{~S}$ requires 323.1836; IR Vmax (solid/cm¹) 2975 (C-H), 2958 (C-H), 2831 (C-H), 1607, 1583, 1411. 


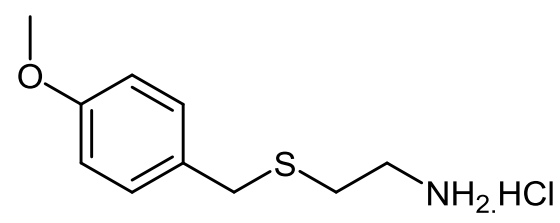

Following procedure for 7 , leaving the reaction mixture overnight gave 8. $\mathrm{mp}$ 136.9-138.3 ${ }^{\circ} \mathrm{C} ;{ }^{1} \mathrm{H}$ NMR $(600 \mathrm{MHz}, \mathrm{MeOD}) \delta 7.27(2 \mathrm{H}, \mathrm{d}, \mathrm{J}=8.7, \mathrm{ArH}), 6.87(2 \mathrm{H}, \mathrm{d}, J=8.7, \mathrm{ArH}), 3.77$ $\left(3 \mathrm{H}, \mathrm{s}, \mathrm{OCH}_{3}\right), 3.74\left(2 \mathrm{H}, \mathrm{s}, \mathrm{ArCH}_{2}\right), 3.01\left(2 \mathrm{H}, \mathrm{t}, J=6.9, \mathrm{CH}_{2} \mathrm{~N}\right), 2.68(2 \mathrm{H}, \mathrm{t}, J=6.9$, $\left.\mathrm{SCH}_{2} \mathrm{CH}_{2}\right) ;{ }^{13} \mathrm{C}$ NMR $(150 \mathrm{MHz}, \mathrm{MeOD}) \delta 160.5,131.2,131.0,115.1,55.7,39.7,35.9$, 29.1; LRMS $(\mathrm{Cl}) \mathrm{m} / \mathrm{z} 198\left(\mathrm{M}^{+} \mathrm{H}^{+}\right), 121\left(\mathrm{PMB}^{+}\right)$. Data in accordance with the literature. ${ }^{3}$

\section{(9H-Fluoren-9-yl)methyl (2-fluoro-2-oxoethyl)carbamate (FMoc-Gly-F) ${ }^{4}$}

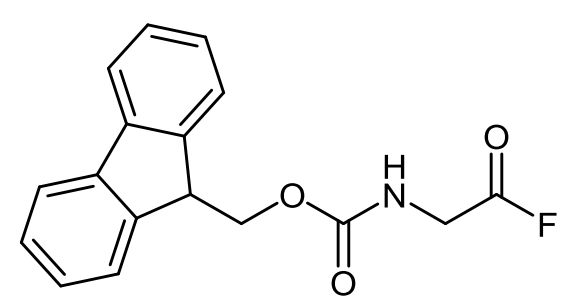

Using flame dried glassware and under an argon atmosphere, to a solution of Fmoc-Gly$\mathrm{OH}(2.973 \mathrm{~g}, 10.0 \mathrm{mmol})$ in anhydrous $\mathrm{CH}_{2} \mathrm{Cl}_{2}(50 \mathrm{~mL})$ and anhydrous THF $(15 \mathrm{~mL})$ was added pyridine $(810 \mu \mathrm{L}, 10 \mathrm{mmol})$ followed by the dropwise addition of cyanuric fluoride $(1.72 \mathrm{~mL}, 20 \mathrm{mmol})$. The reaction was stirred overnight. The precipitate and yellow solution were washed with ice water $(2 \times 50 \mathrm{~mL})$ and then brine $(50 \mathrm{~mL})$. The organic layer was reduced in vacuo. Addition of $\mathrm{CH}_{2} \mathrm{Cl}_{2}$ and hexane precipitated FMoc-Gly-F as a yellow solid (2.5903 g, $8.654 \mathrm{mmol}, 87 \%)$; $\mathbf{R f} 0.26$ (1:9 MeOH:CHCl 3 ); mp 127.5-128.4 ${ }^{\circ} \mathrm{C}$ (lit. 134-135 $\left.{ }^{\circ} \mathrm{C}^{14}\right) ;{ }^{1} \mathrm{H}$ NMR $\left(600 \mathrm{MHz}, \mathrm{CDCl}_{3}\right) \delta 7.77(2 \mathrm{H}, \mathrm{d}, J=7.6, \mathrm{ArH}), 7.59(2 \mathrm{H}, \mathrm{d}, J=$ 7.4, ArH), $7.41(2 \mathrm{H}, \mathrm{td}, J=7.4,1.4, \mathrm{ArH}), 7.32(2 \mathrm{H}, \mathrm{td}, J=7.5,1.0, \mathrm{ArH}), 5.24(1 \mathrm{H}, \mathrm{br} \mathrm{s}$, $\mathrm{NH}), 4.47(2 \mathrm{H}, \mathrm{d}, J=6.9, \mathrm{FmocCH})_{2}, 4.24(1 \mathrm{H}, \mathrm{t}, J=6.9, \mathrm{FmocCHCH}), 4.18(2 \mathrm{H}, \mathrm{m}$, $\left.\mathrm{NHCH}_{2}\right) ;{ }^{13} \mathrm{C}$ NMR $\left(150 \mathrm{MHz}, \mathrm{CDCl}_{3}\right) \delta 160.7\left(\mathrm{~d}, \mathrm{~J}_{\mathrm{C}-\mathrm{F}}=363 \mathrm{~Hz}\right), 156.1,143.6,141.4$, 127.9, 127.2, 125.0, 120.1, 67.6, 47.1, 41.2 (d, JC-F $=87 \mathrm{~Hz})$; IR Umax $3333(\mathrm{~N}-\mathrm{H}), 3212$, 
$2970(\mathrm{C}-\mathrm{H}), 1865,1840$ (C=O), 1678 (C=O), 1534, 1262, 734 (C-F).

$N$-(2-((4-Methoxybenzyl)thio)-1-(4,4,5,5-tetramethyl-1,3,2-dioxaborolan-2-

yl)ethyl)acetamide (9a)

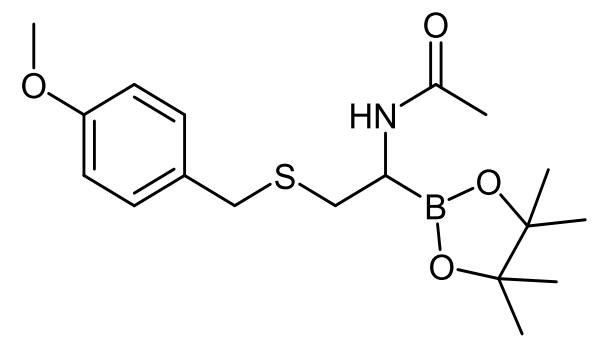

Acetyl chloride ( $65 \mu \mathrm{L}, 0.42 \mathrm{mmol}$ ) was added to a solution of 7 (30 mg, $84 \mu \mathrm{mol}$ ) in acetonitrile $(1 \mathrm{~mL})$ followed by pyridine $(40 \mu \mathrm{L}, 0.50 \mathrm{mmol})$. The reaction was stirred for 18 h. Solvent was removed in vacuo and EtOAc $(3 \mathrm{~mL})$ added. The reaction mixture was filtered and the filtrate washed with $\mathrm{H}_{2} \mathrm{O}(3 \mathrm{~mL})$, phosphoric acid $\left(1 \%\right.$ in $\left.\mathrm{H}_{2} \mathrm{O}, 3 \mathrm{~mL}\right)$, potassium carbonate $\left(2 \%\right.$ in $\left.\mathrm{H}_{2} \mathrm{O}, 3 \mathrm{~mL}\right)$ and then brine $(3 \mathrm{~mL})$. All aqueous layers were back extracted with EtOAc. Removal of solvent in vacuo gave an orange oil which was purified by column chromatography (20\% EtOAc in $\mathrm{CH}_{2} \mathrm{Cl}_{2}$ ) to give 9 a as an orange oil (6.7 mg, 22\%). ${ }^{1} \mathrm{H}$ NMR $\left(\mathrm{CDCl}_{3}, 600 \mathrm{MHz}\right) 7.21(2 \mathrm{H}, \mathrm{d}, J=8.6, \mathrm{ArH}), 7.00(1 \mathrm{H}, \mathrm{br} \mathrm{s}, \mathrm{NH}), 6.83$ $(2 \mathrm{H}, \mathrm{d}, J=8.6, \mathrm{ArH}), 3.78\left(3 \mathrm{H}, \mathrm{s}, \mathrm{OCH}_{3}\right), 3.66\left(1 \mathrm{H}, \mathrm{d}, J=13.6,1 \times \mathrm{ArCH}_{2}\right), 3.63(1 \mathrm{H}, \mathrm{d}, J$ $\left.=13.6,1 \times \mathrm{ArCH}_{2}\right), 2.81\left(1 \mathrm{H}, \mathrm{dd}, J=14.3,2.8,1 \times \mathrm{CH}_{2} \mathrm{CH}\right), 2.65(1 \mathrm{H}, \mathrm{br} \mathrm{d}, J=12.2$, CHB), $2.45\left(1 \mathrm{H}, \mathrm{dd}, J=14.3,12.8,1 \times \mathrm{CH}_{2} \mathrm{CH}\right), 2.05\left(3 \mathrm{H}, \mathrm{s}, \mathrm{C}(\mathrm{O}) \mathrm{CH}_{3}\right), 1.16(6 \mathrm{H}, \mathrm{s}, 2 \times$ pinacol- $\left.\mathrm{CH}_{3}\right), 1.15\left(6 \mathrm{H}, \mathrm{s}, 2 \times\right.$ pinacol- $\left.\mathrm{CH}_{3}\right) ;{ }^{13} \mathrm{C} \mathrm{NMR}\left(\mathrm{CDCl}_{3}, 150 \mathrm{MHz}\right) \delta 176.0,158.8$, 130.7, 130.1, 114.1, 80.4, 55.4, 44.4, 35.2, 34.2, 25.3, 24.9, 18.1; LRMS (Cl) 366 (40\%, $\left.[\mathrm{M}+\mathrm{H}]^{+}\right), 266(51 \%), 243(33 \%), 212\left(57 \%,[\mathrm{M}-\mathrm{SPMB}]^{+}\right), 121\left(100 \%, \mathrm{PMB}^{+}\right)$; HRMS Found 366.19151, $\mathrm{C}_{18} \mathrm{H}_{28} \mathrm{BNO}_{4} \mathrm{~S}$ requires 366.19103; IR $v_{\max }\left(\mathrm{film} / \mathrm{cm}^{-1}\right) 3260(\mathrm{~N}-\mathrm{H})$, 2960(C-H), 2924 (C-H), 2853 (C-H), 1609 (C=O), 1511. 

yl)ethyl)acetamide (9b)<smiles>COc1ccc(CSCC(NC(=O)CCl)B2OC(C)(C)C(C)(C)O2)cc1</smiles>

Using flame dried glassware and under an argon atmosphere, a solution of $7(82.3 \mathrm{mg}$, $0.193 \mathrm{mmol})$ in anhydrous $\mathrm{CH}_{2} \mathrm{Cl}_{2}(1 \mathrm{~mL})$ was cooled to $0{ }^{\circ} \mathrm{C}$. Chloroacetyl chloride $(17 \mu \mathrm{L}$, $0.231 \mathrm{mmol}$ ) was added followed by the dropwise addition of NMM $(30 \mu \mathrm{L}, 0.270 \mathrm{mmol})$. The solution was allowed to warm to room temperature and stirred for $90 \mathrm{~min}$. The reaction was cooled to $0{ }^{\circ} \mathrm{C}$ and $\mathrm{HCl}(0.2 \mathrm{M}, 7 \mathrm{~mL})$ added. The reaction mixture was diluted with EtOAc and the organic layer was separated, dried $\left(\mathrm{MgSO}_{4}\right)$, filtered and concentrated in vacuo to give a brown oil. On addition of $\mathrm{Et}_{2} \mathrm{O}$ a brown solid formed which was filtered. The filtrate was concentrated in vacuo to give $9 \mathrm{~b}$ as a brown oil $(65.4 \mathrm{mg}$, $85 \%) .{ }^{1} \mathrm{H}$ NMR $\left(\mathrm{CDCl}_{3}, 600 \mathrm{MHz}\right) \delta 7.66(1 \mathrm{H}, \mathrm{br} \mathrm{s}, \mathrm{NH}), 7.21(2 \mathrm{H}, \mathrm{d}, J=8.7, \mathrm{ArH}), 6.83$ $(2 \mathrm{H}, \mathrm{d}, J=8.7, \mathrm{ArH}), 4.15\left(2 \mathrm{H}, \mathrm{s}, \mathrm{CH}_{2} \mathrm{Cl}\right), 3.78\left(3 \mathrm{H}, \mathrm{s}, \mathrm{OCH}_{3}\right), 3.67\left(2 \mathrm{H}, \mathrm{s}, \mathrm{ArCH}_{2}\right), 2.97$ $(1 \mathrm{H}, \mathrm{br} d, J=11.1, \mathrm{CHB}), 2.76\left(1 \mathrm{H}, \mathrm{dd}, J=14.0,3.0,1 \times \mathrm{CH}_{2} \mathrm{CHB}\right), 2.52(1 \mathrm{H}, \mathrm{dd}, J=14.0$, 11.6, $\left.1 \times \mathrm{CH}_{2} \mathrm{CHB}\right), 1.21\left(6 \mathrm{H}, \mathrm{s}, 2 \times\right.$ pinacol- $\left.\mathrm{CH}_{3}\right), 1.19\left(6 \mathrm{H}, \mathrm{s}, 2 \times\right.$ pincaol- $\left.\mathrm{CH}_{3}\right) ;{ }^{13} \mathrm{C} \mathrm{NMR}$ $\left(\mathrm{CDCl}_{3}, 150 \mathrm{MHz}\right) \delta 171.1,158.8,130.1(2 \mathrm{C}), 114.1,82.5,55.4,41.6$ (br), 41.3, 35.4, 33.0, 24.9, 24.63; LRMS (Cl) $402\left(41 \%,[\mathrm{M}+\mathrm{H}]^{+}, \mathrm{Cl}^{37}\right), 404\left(100 \%,[\mathrm{M}+\mathrm{H}]^{+}, \mathrm{Cl}{ }^{35}\right)$; HRMS Found 399.1552, $\mathrm{C}_{18} \mathrm{H}_{28} \mathrm{BCINO}{ }_{4} \mathrm{~S}$ requires 399.1551; IR $v_{\max }\left(\mathrm{film} / \mathrm{cm}^{-1}\right) 2923(\mathrm{C}-\mathrm{H}), 1733(\mathrm{C}=\mathrm{O})$, 1661, 1593, 1494, 1434. 
tert-Butyl (2-((2-((4-methoxybenzyl)thio)-1-(4,4,5,5-tetramethyl-1,3,2-dioxaborolan-2yl)ethyl)amino)-2-oxoethyl)carbamate (10a)

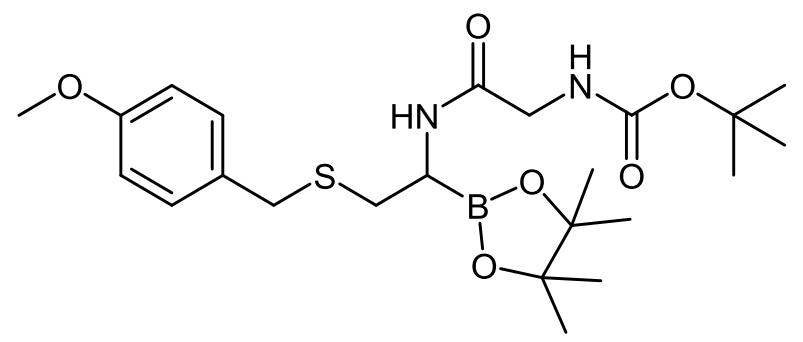

Using flame dried glassware under an argon atmosphere, Boc-Gly-OH $(87.5 \mathrm{mg}, 0.50$ mmol) was dissolved in anhydrous $\mathrm{CH}_{2} \mathrm{Cl}_{2}(1.5 \mathrm{~mL})$ and cooled to $-20{ }^{\circ} \mathrm{C}$. NMM $(66 \mu \mathrm{L}$, $0.60 \mathrm{mmol}$ ) was added followed by IBCF $(58 \mu \mathrm{L}, 0.45 \mathrm{mmol})$ and the reaction stirred for 5 h at $-20{ }^{\circ} \mathrm{C}$. $\mathrm{HCl}$ salt $23(23.4 \mathrm{mg}, 65.1 \mu \mathrm{mol})$ was added followed by NMM $(7 \mu \mathrm{L}, 65 \mu \mathrm{mol})$ and the reaction allowed to warm to it and stirred for $18 \mathrm{~h}$. The reaction mixture was concentrated in vacuo and the resultant oil purified by column chromatography $(2 \% \mathrm{MeOH}$ in EtOAc) using deactivated silica $\left(35 \% \mathrm{H}_{2} \mathrm{O} \mathrm{w} / \mathrm{w}\right)$ to give 36 as a pale yellow oil $(27.9 \mathrm{mg}$, 89\%). ${ }^{1} \mathrm{H}$ NMR $\left(\mathrm{CDCl}_{3}, 600 \mathrm{MHz}\right) \delta 7.51(1 \mathrm{H}, \mathrm{br} \mathrm{s}, \mathrm{CHNH}), 7.21(2 \mathrm{H}, \mathrm{d}, J=8.7, \mathrm{ArH}), 6.82$ $(2 \mathrm{H}, \mathrm{d}, J=8.7, \mathrm{ArH}), 5.29\left(1 \mathrm{H}, \mathrm{br} \mathrm{s}, \mathrm{CH}_{2} \mathrm{NH}\right), 3.93\left(2 \mathrm{H}, \mathrm{d}, J=5.7, \mathrm{NHCH}_{2}\right), 3.78(3 \mathrm{H}, \mathrm{s}$, $\left.\mathrm{OCH}_{3}\right), 3.65\left(2 \mathrm{H}, \mathrm{s}, \mathrm{ArCH}_{2}\right), 2.81(1 \mathrm{H}, \mathrm{br} \mathrm{d}, J=11.5, \mathrm{CHB}), 2.75(1 \mathrm{H}, \mathrm{dd}, J=14.1,3.2,1 \times$ $\left.\mathrm{SCH}_{2} \mathrm{CH}\right), 2.46\left(1 \mathrm{H}, \mathrm{dd}, J=14.1,11.5,1 \times \mathrm{SCH}_{2} \mathrm{CH}\right), 1.44(9 \mathrm{H}, \mathrm{s}, \mathrm{Bu}), 1.18(6 \mathrm{H}, \mathrm{s}, 2 \times$ pinacol- $\left.\mathrm{CH}_{3}\right), 1.16\left(6 \mathrm{H}, \mathrm{s}, 2 \times\right.$ pinacol- $\left.\mathrm{CH}_{3}\right) ;{ }^{13} \mathrm{C} \mathrm{NMR}\left(\mathrm{CDCl}_{3}, 150 \mathrm{MHz}\right) \delta 174.9,158.7$, $130.3,130.1,114.1,81.6,55.4,54.0,41.4,35.2,33.6,29.8,28.4,25.0,24.9,14.3$; LRMS (Cl) $481\left(100 \%,[\mathrm{M}+\mathrm{H}]^{+}\right)$; HRMS Found 480.2575, $\mathrm{C}_{23} \mathrm{H}_{37} \mathrm{BN}_{2} \mathrm{O}_{6} \mathrm{~S}$ requires 480.2574; IR (film/cm-1) 2970 (C-H), 2926 (C-H), 1697 (br, C=O), 1609 (C=O), 1511, 1456. 


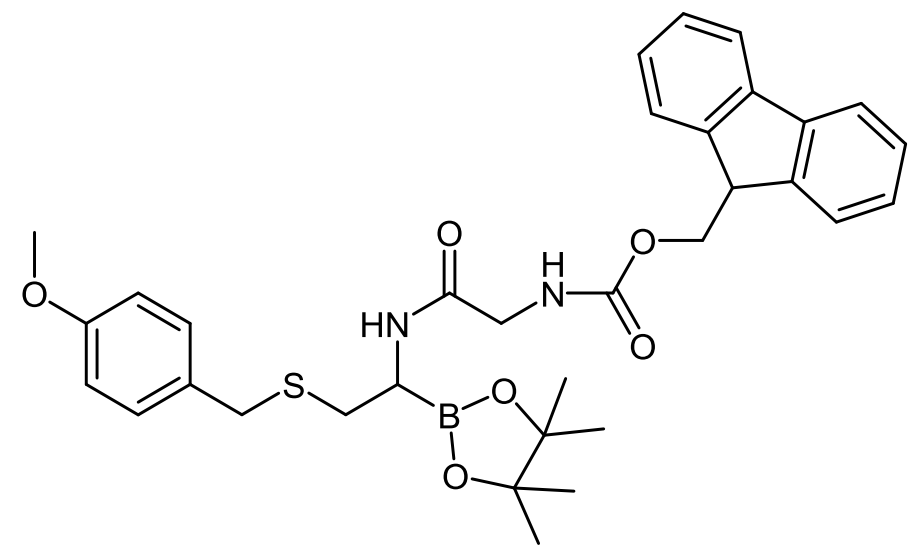

Using flame dried glassware and under an argon atmosphere FMoc-Gly-F (15.9 mg, 53.0 $\mu \mathrm{mol})$ was added to a solution of $\mathrm{HCl}$ salt $7(15.9 \mathrm{mg}, 44.2 \mu \mathrm{mol})$ in anhydrous $\mathrm{CH}_{2} \mathrm{Cl}_{2}(1.5$ $\mathrm{mL}$ ) followed by DIPEA $(15 \mu \mathrm{L}, 88 \mu \mathrm{mol})$. The reaction was stirred for $18 \mathrm{~h}$. The solution was washed with $\mathrm{HCl}(2 \mathrm{M}, 2 \times 3 \mathrm{~mL}), \mathrm{H}_{2} \mathrm{O}(2 \times 3 \mathrm{~mL})$, sodium bicarbonate $(2 \times 3 \mathrm{~mL})$ and $\mathrm{H}_{2} \mathrm{O}(2 \times 3 \mathrm{~mL})$. All aqueous layers were back extracted with $\mathrm{CH}_{2} \mathrm{Cl}_{2}$. The combined organic layers were dried $\left(\mathrm{MgSO}_{4}\right)$, filtered and concentrated in vacuo to give an orange residue which was purified by column chromatography $\left(1 \% \mathrm{MeOH}\right.$ in $\left.\mathrm{CHCl}_{3}\right)$ using deactivated silica $\left(35 \% \mathrm{H}_{2} \mathrm{O} \mathrm{w} / \mathrm{w}\right)$ to give 39 as an orange gum (16.0 mg, 60\%). ${ }^{1} \mathrm{H}$ NMR $\left(\mathrm{CDCl}_{3}, 600 \mathrm{MHz}\right) \delta 7.77(2 \mathrm{H}, \mathrm{d}, J=7.6, \mathrm{FmocH}), 7.58(2 \mathrm{H}, \mathrm{d}, J=7.4, \mathrm{FmocH}), 7.40(2 \mathrm{H}$, $\mathrm{t}, J=7.6, \mathrm{FmocH}), 7.31(2 \mathrm{H}, \mathrm{t}, J=7.4, \mathrm{FmocH}), 7.18(2 \mathrm{H}, \mathrm{d}, J=8.6, \mathrm{ArH}), 7.03(1 \mathrm{H}, \mathrm{br} \mathrm{s}$, $\mathrm{CHNH}), 6.79(2 \mathrm{H}, \mathrm{d}, J=8.6, \mathrm{ArH}), 5.33\left(1 \mathrm{H}, \mathrm{br} \mathrm{s}, \mathrm{CH}_{2} \mathrm{NH}\right), 4.46(2 \mathrm{H}, \mathrm{d}, J=6.7$, $\left.\mathrm{FmocCH}_{2}\right), 4.22\left(1 \mathrm{H}, \mathrm{d}, J=6.7, \mathrm{FmocCH}_{2} \mathrm{CH}\right), 3.98\left(1 \mathrm{H}, \mathrm{dd}, J=17.3,5.8,1 \times \mathrm{CH}_{2} \mathrm{NH}\right)$, $3.92\left(1 \mathrm{H}, \mathrm{dd}, J=17.3,5.7,1 \times \mathrm{CH}_{2} \mathrm{NH}\right), 3.74\left(3 \mathrm{H}, \mathrm{s}, \mathrm{OCH}_{3}\right), 3.63\left(2 \mathrm{H}, \mathrm{s}, \mathrm{ArCH}_{2} \mathrm{~S}\right), 2.89$ $(1 \mathrm{H}, \mathrm{br} d, J=10.0, \mathrm{CHB}), 2.80\left(1 \mathrm{H}, \mathrm{dd}, J=13.7,3.4,1 \times \mathrm{CH}_{2} \mathrm{CHB}\right), 2.50(1 \mathrm{H}, \mathrm{dd}, J=$ 13.7, 11.6, $\left.1 \times \mathrm{CH}_{2} \mathrm{CHB}\right), 1.20\left(6 \mathrm{H}, \mathrm{s}, 2 \times\right.$ pinacol- $\left.\mathrm{CH}_{3}\right), 1.19\left(6 \mathrm{H}, \mathrm{s}, 2 \times\right.$ pinacol- $\left.\mathrm{CH}_{3}\right) ;{ }^{13} \mathrm{C}$ NMR $\left(\mathrm{CDCl}_{3}, 150 \mathrm{MHz}\right) \delta 173.0,158.7,156.5,143.7,141.4,130.4,130.1,127.9,127.3$, 125.1, 120.2, 114.1, 82.2, 67.4, 55.4, 47.2, 42.3, 41.0 (br), 35.5, 33.7, 25.1, 24.9; LRMS (Cl) $603\left(3 \%,[\mathrm{M}+\mathrm{H}]^{+}\right), 503(3 \%), 381(9 \%), 325(8 \%), 281(7 \%), 207(7 \%), 179(100 \%$, $\left.\left[\mathrm{Fmoc}-\mathrm{CO}_{2}\right]^{+}\right), 147(8 \%), 121\left(36 \%, \mathrm{PMB}^{+}\right), 103$ (17\%); HRMS Found 603.27061, $\mathrm{C}_{33} \mathrm{H}_{39} \mathrm{BN}_{2} \mathrm{O}_{6} \mathrm{~S}$ requires 603.27001; IR $v_{\max }\left(\mathrm{film} / \mathrm{cm}^{-1}\right) 3300(\mathrm{~N}-\mathrm{H}), 2970(\mathrm{C}-\mathrm{H}), 2928$ (C$H), 1711(C=O), 1680(C=O), 1610,1536,1511,1462,1450$. 


\section{(2,2-Diethoxyethyl)(4-methoxybenzyl)sulfane (3b)}
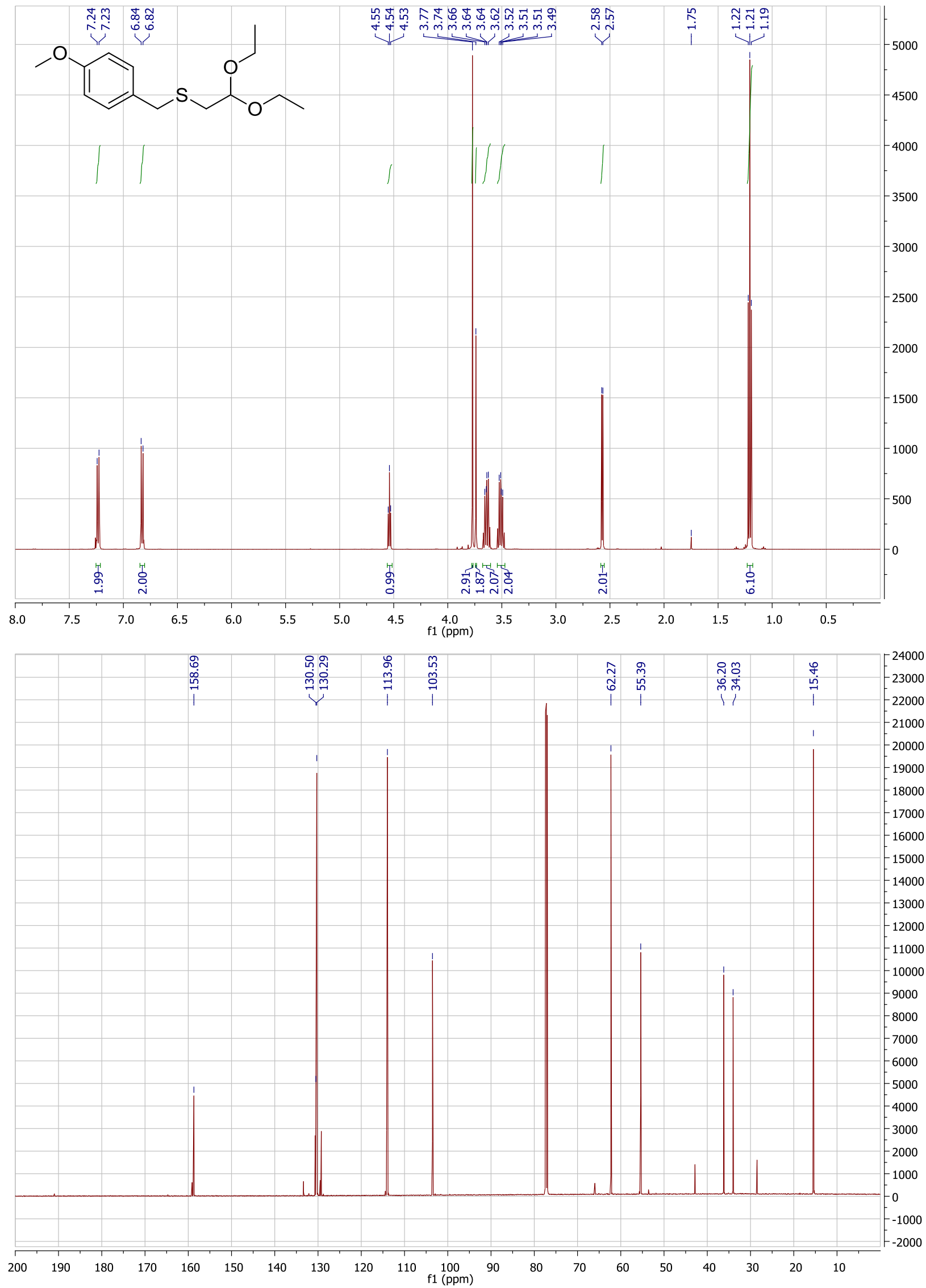
2-((4-methoxybenzyl)thio)acetaldehyde (4)
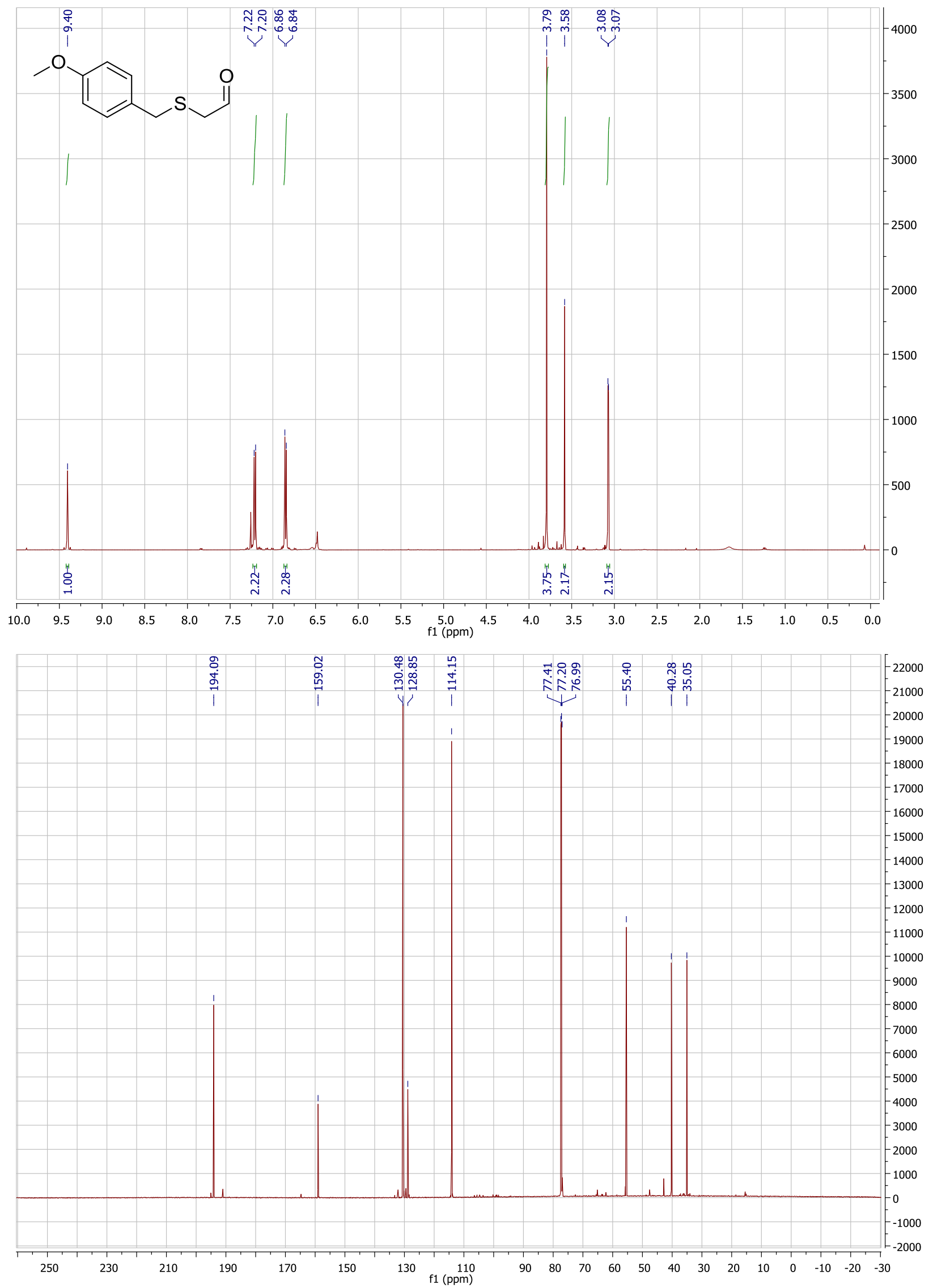
(E)-N-(2-((4-Methoxybenzyl)thio)ethylidene)-2-methylpropane-2-sulfinamide (5)

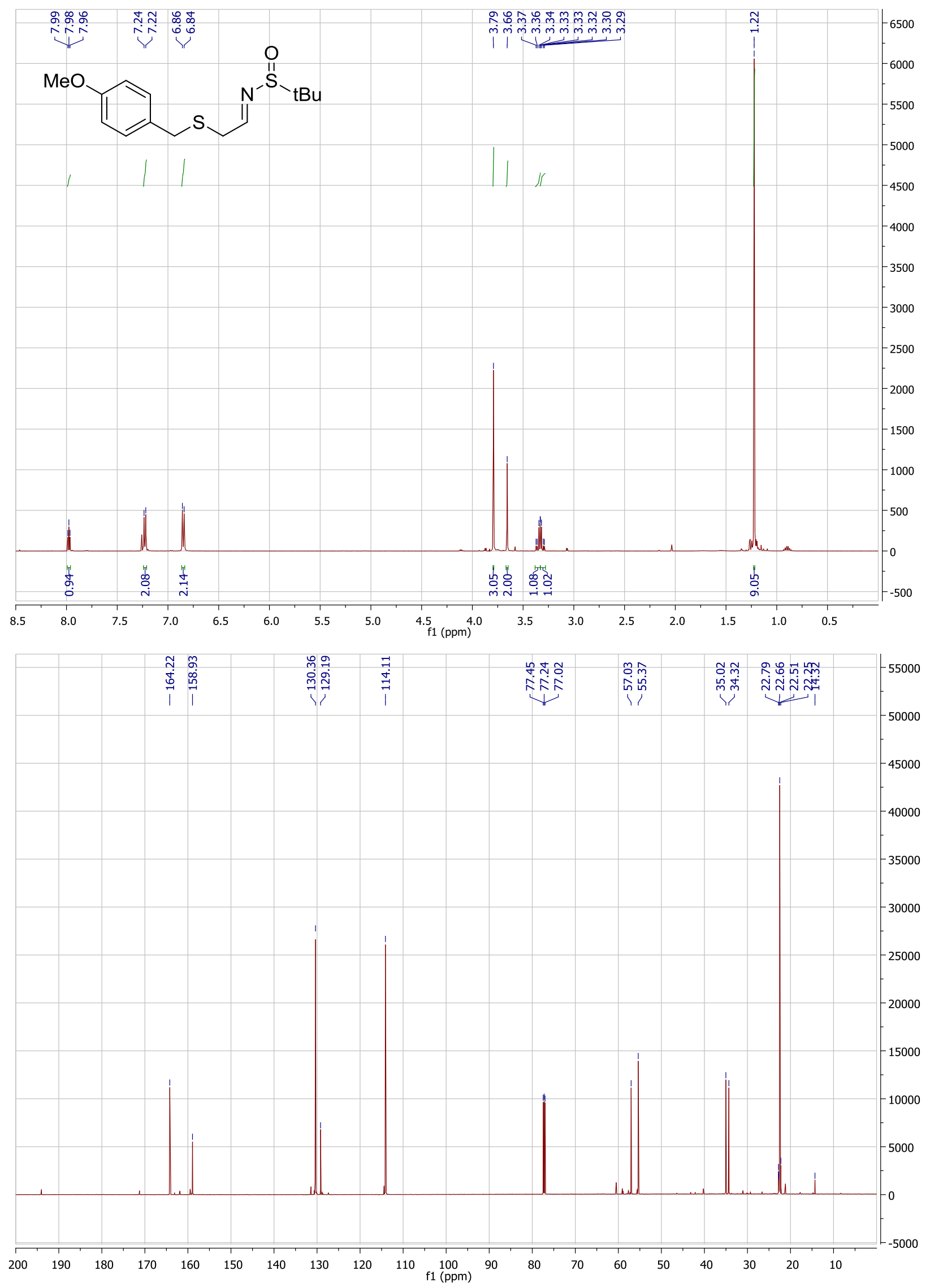


$N$-(2-((4-Methoxybenzyl)thio)-1-(4,4,5,5-tetramethyl-1,3,2-dioxaborolan-2-yl)ethyl)-2-methylpropane-2sulfinamide (6)
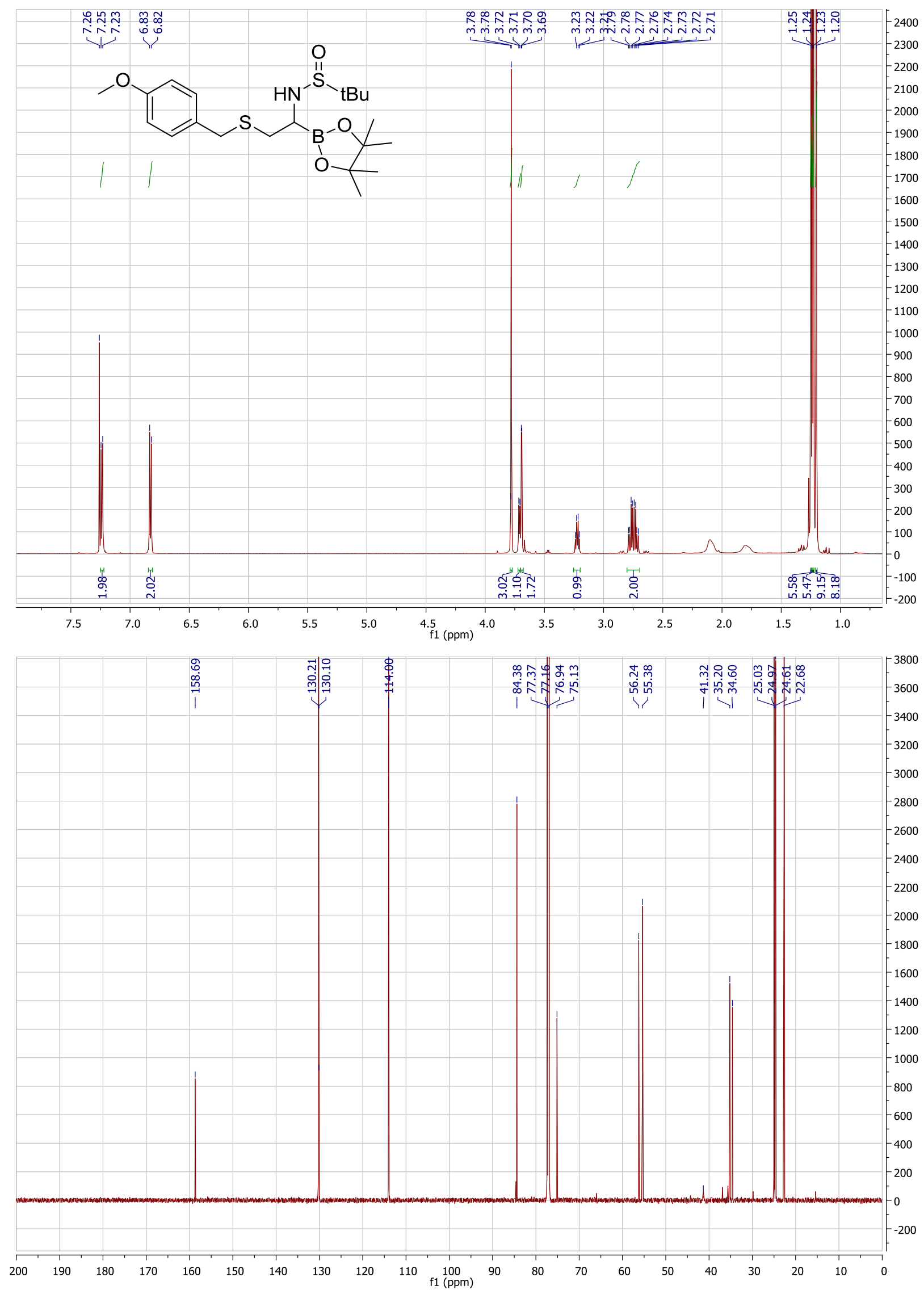
2-((4-Methoxybenzyl)thio)-1-(4,4,5,5-tetramethyl-1,3,2-dioxaborolan-2-yl)ethanamine hydrochloride (7)
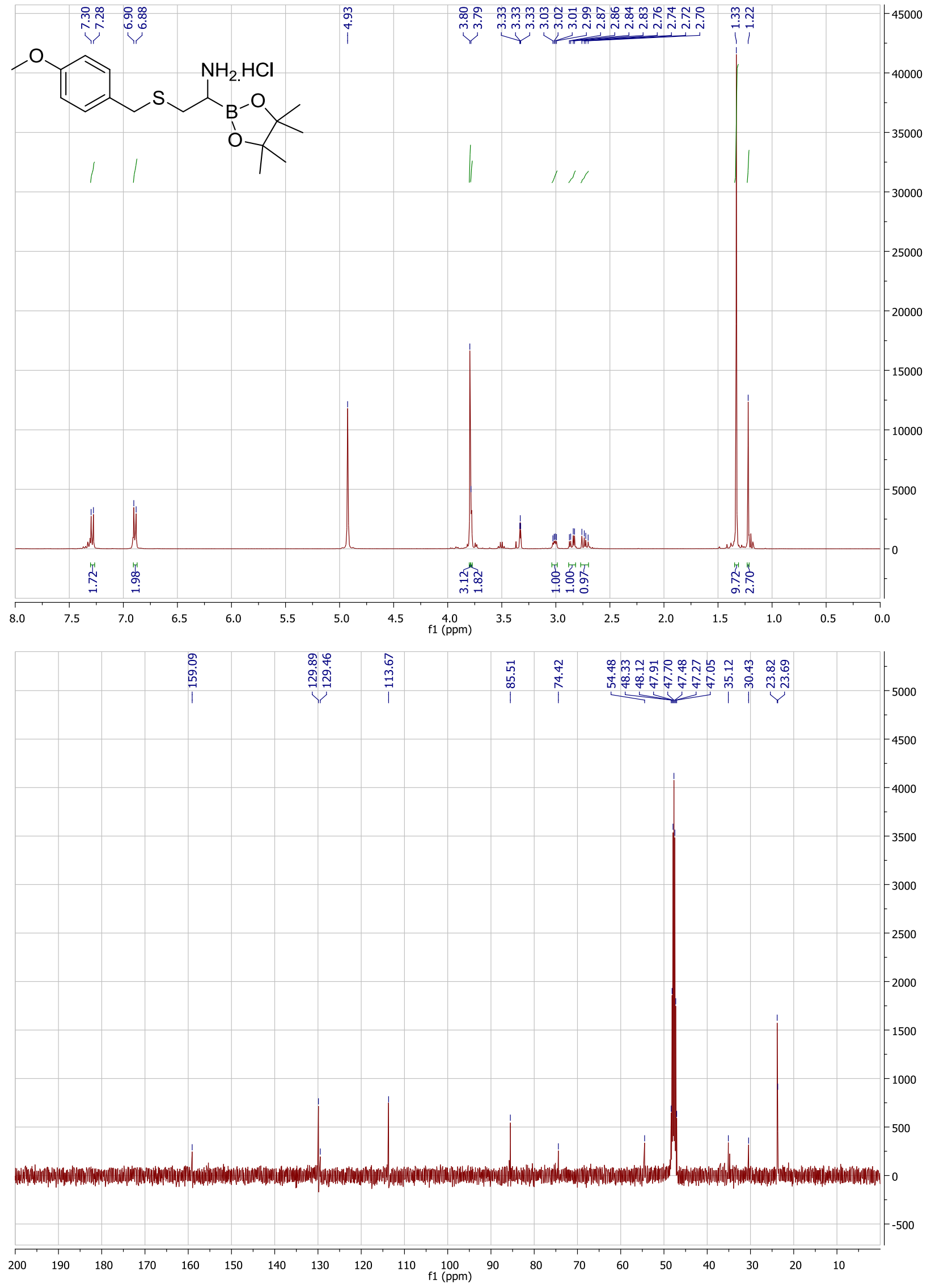
2-((4-Methoxybenzyl)thio)ethanamine hydrochloride (8)
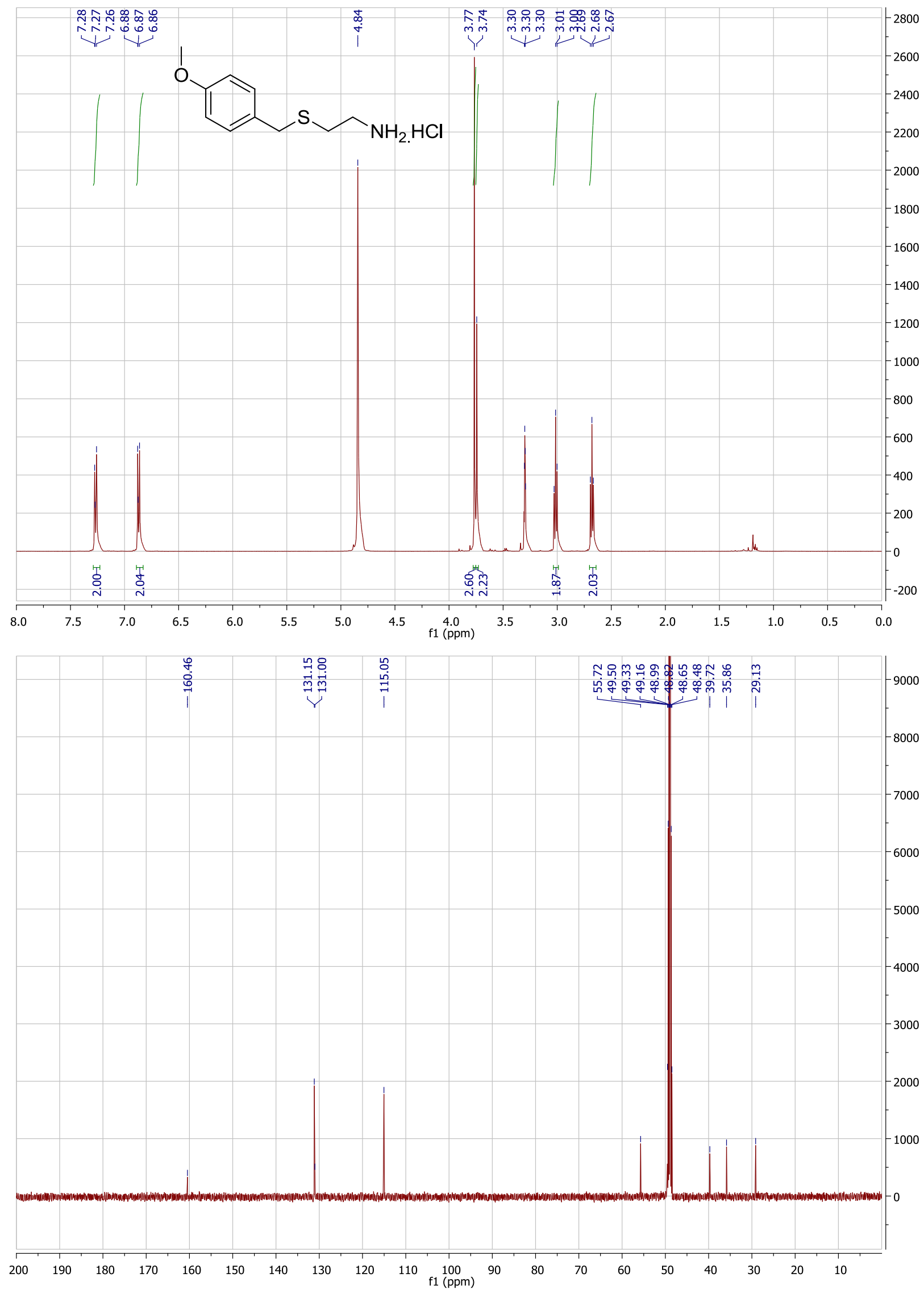
$N$-(2-((4-Methoxybenzyl)thio)-1-(4,4,5,5-tetramethyl-1,3,2-dioxaborolan-2-yl)ethyl)acetamide (9a)
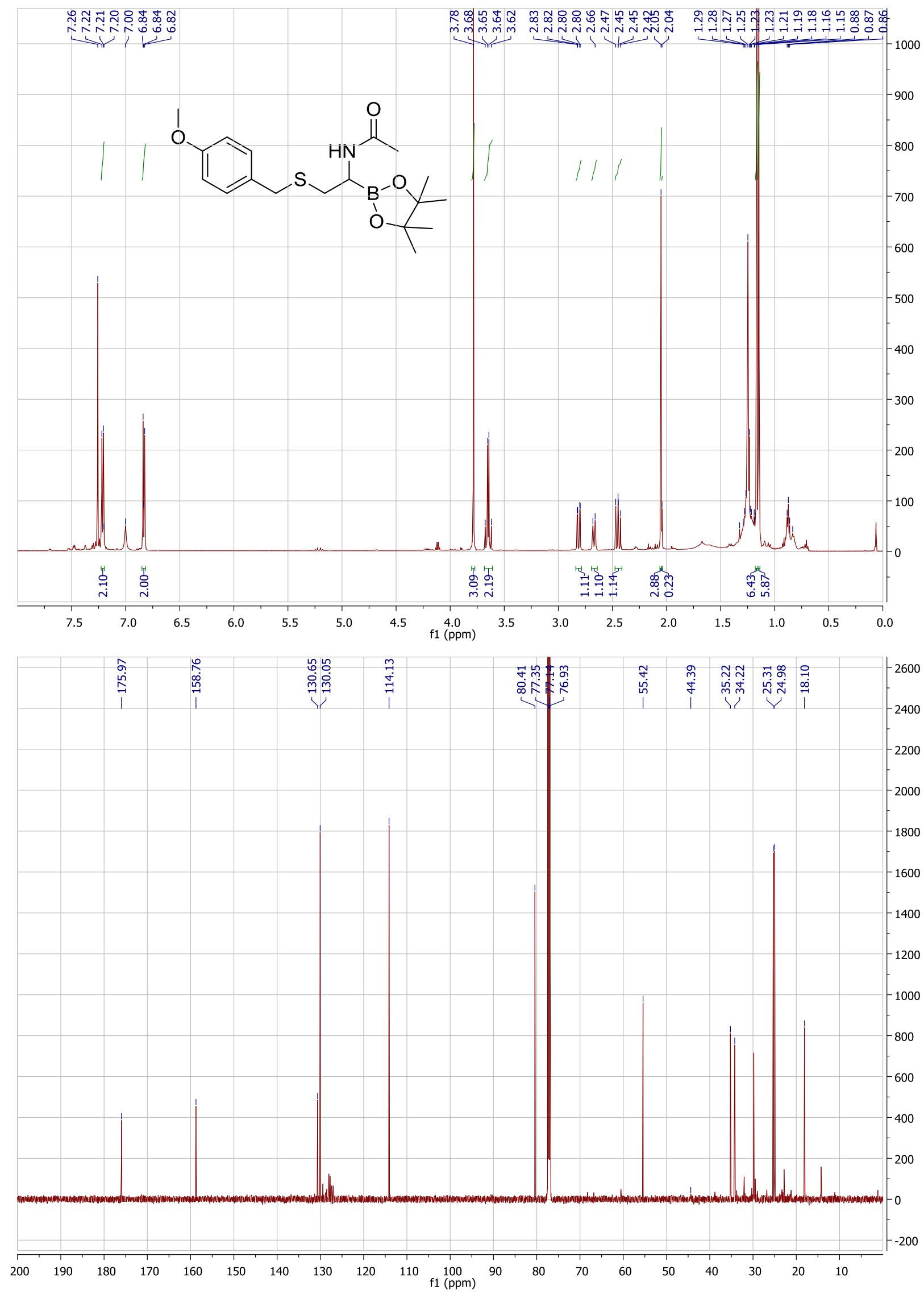
2-Chloro- $N$-(2-((4-methoxybenzyl)thio)-1-(4,4,5,5-tetramethyl-1,3,2-dioxaborolan-2-yl)ethyl)acetamide (9b)
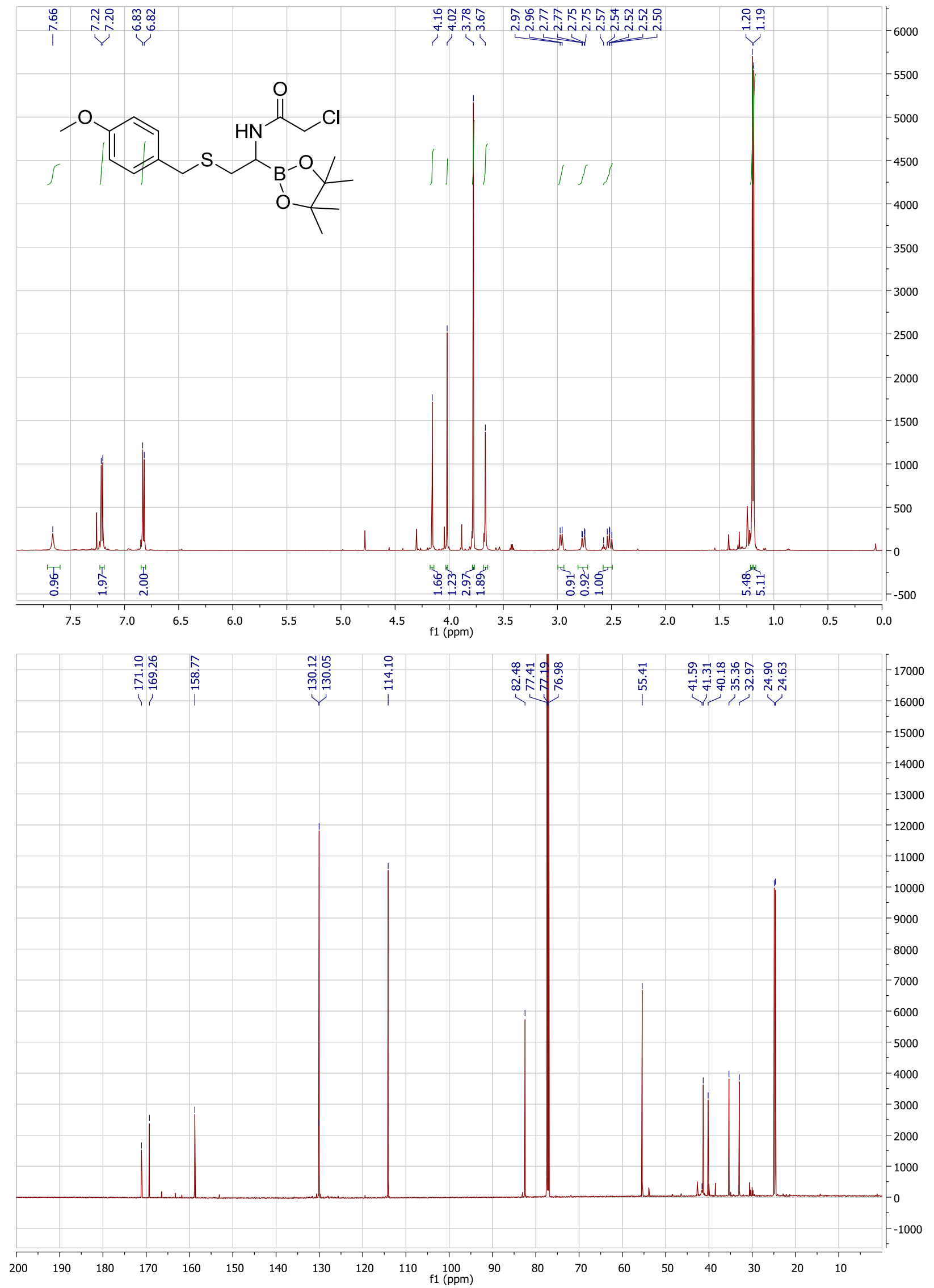
tert-Butyl (2-((2-((4-methoxybenzyl)thio)-1-(4,4,5,5-tetramethyl-1,3,2-dioxaborolan-2-yl)ethyl)amino)-2oxoethyl)carbamate (10a)
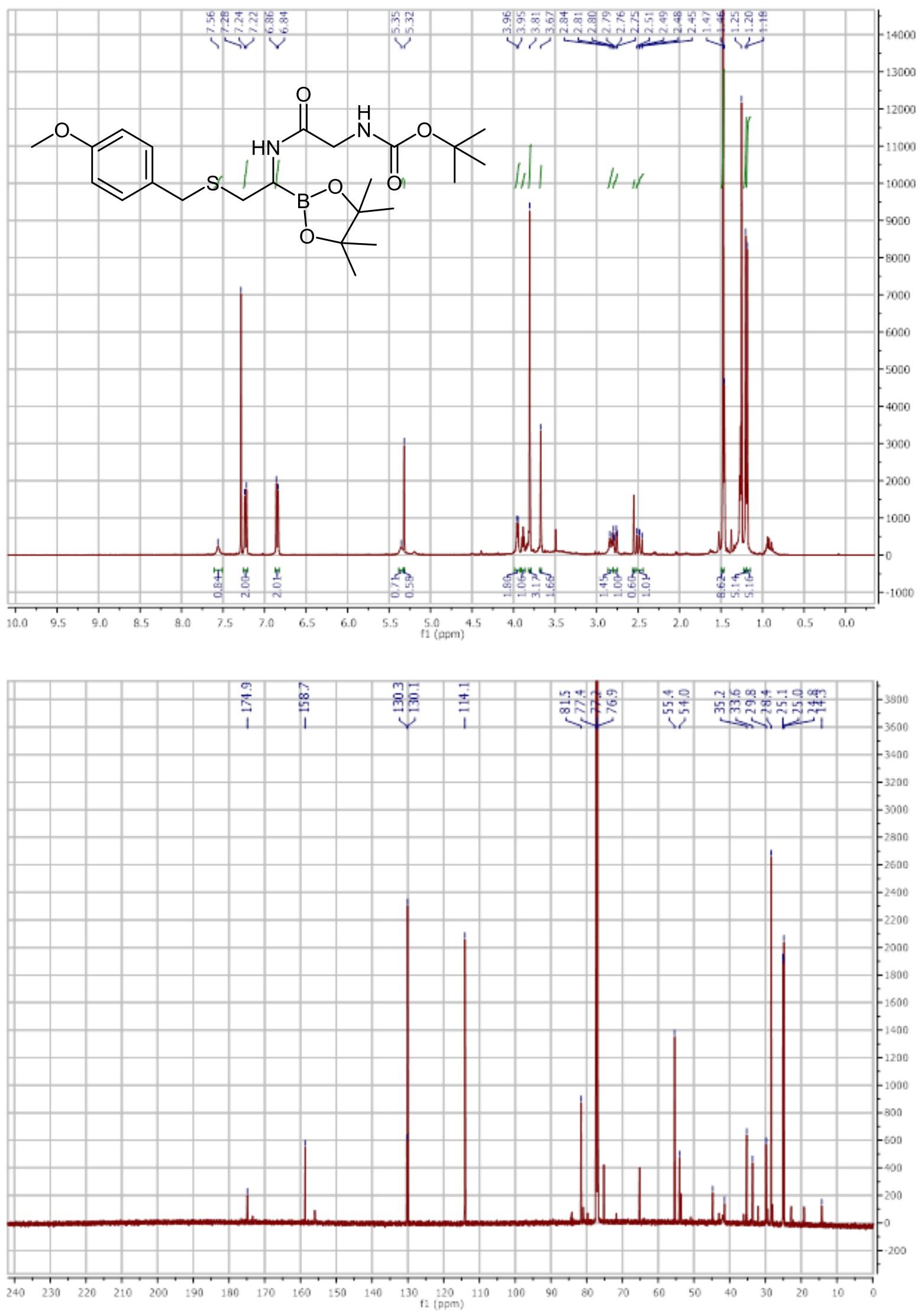
(9H-Fluoren-9-yl)methyl(2-((2-((4-methoxybenzyl)thio)-1-(4,4,5,5-tetramethyl-1,3,2-dioxaborolan-2yl)ethyl)amino)-2-oxoethyl)carbamate (10b)
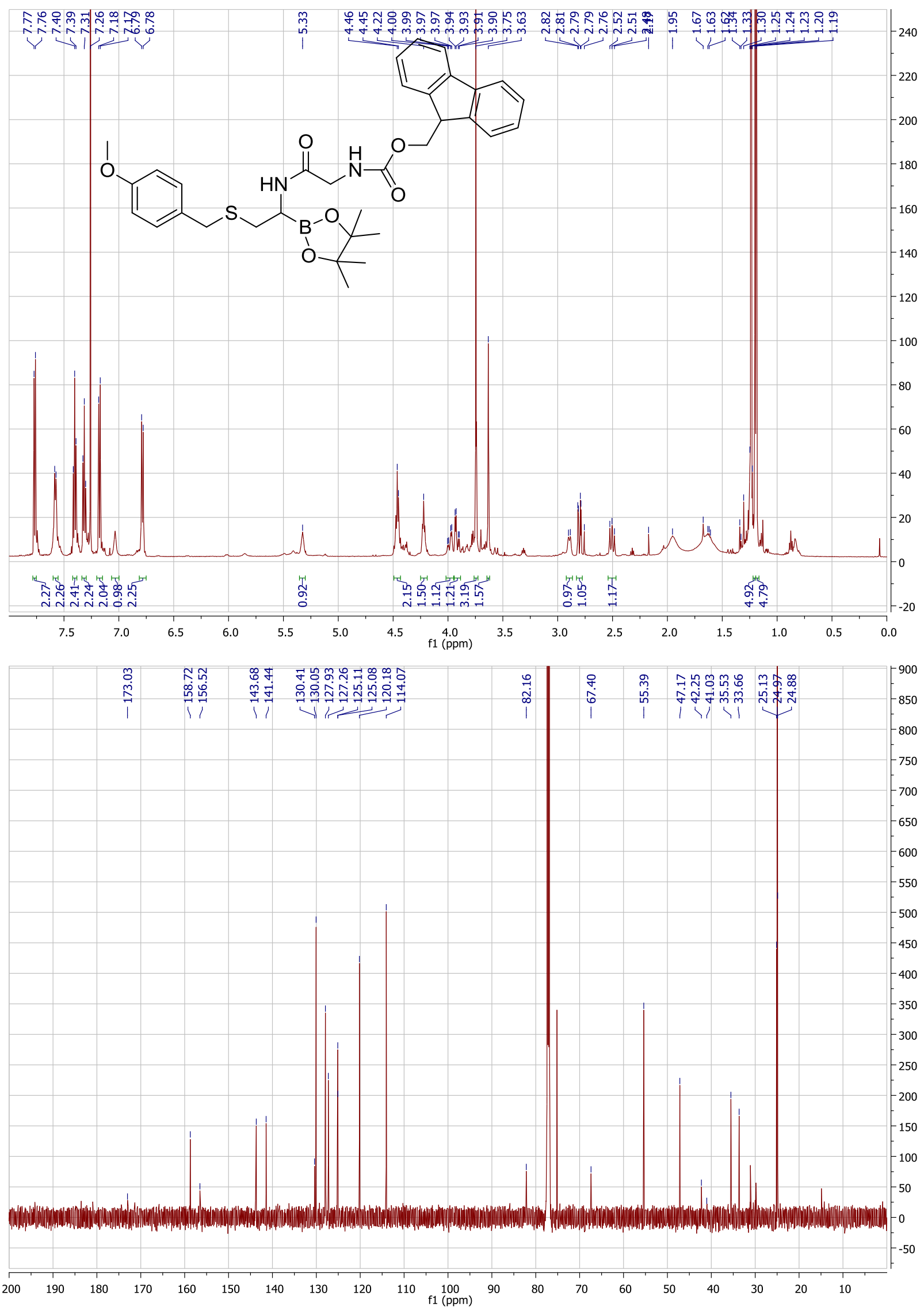


\section{References}

(1) Lantos, I.; Razgaitis, C.; Sutton, B. M. J. Heterocycl. Chem. 1982, 19, 1375.

(2) Yoneda, K.; Ota, A.; Kawashima, Y. Chem. Pharm. Bull. 1993, 41, 876

(3) Ghosh, S.; Tochtrop, G. P. Tetrahedron Lett. 2009, 50, 1723.

(4) Bertho, J.-N; Loffet, A.; Pinel, C.; Reuther, F.; Sennyey, G. Tetrahedron Lett. 1991, 32, 1303. 